\title{
EL GOBIERNO ELECTRÓNICO Y LOS SERVICIOS PÚBLICOS DEL MARCO LEGAL ESPAÑOL
}

\section{The Electronic Government and Public Services. Legal Framework Spanish}

\author{
Jorge López Córdova'
}

\section{RESUMEN}

El objetivo de este trabajo es proporcionar una visión bastante clara acerca de los beneficios que brinda la implantación de las nuevas tecnologías de la información y las comunicaciones (TIC) en la administración pública: el denominado gobierno electrónico. Estos beneficios casi siempre se traducen en servicios electrónicos a los ciudadanos y a las empresas e instituciones gubernamentales, e inciden en el acompañamiento de un cambio organizativo con nuevos métodos para mejorar los servicios públicos, las políticas públicas y los procesos democráticos que pautan nuevas formas de relación. El gobierno electrónico supone un nuevo modelo de desarrollo, lo que demanda la creación de nuevas reglas para acompasar tan profundos cambios. También, haremos referencia al marco jurídico en el que debe basarse la acción pública en la actualidad y la lentitud de los mecanismos legales establecidos para su adecuación al contexto cambiante. Estos parecen ser elementos muy importantes de bloqueo para los proyectos de gobierno electrónico.

Palabras clave: Gobierno electrónico, administración electrónica, servicios electrónicos, modernización gubernamental

\begin{abstract}
The aim of this paper is to provide insight about the benefits offered by the implementation of new Information Technology and Communications (ICT) in public administration, the so-called Electronic Government; providing electronic services to the citizens, businesses and government institutions; accompanied by organizational change and new methods to improve public services, public policies and democratic processes, which guide new forms of relationship, and a new development model, which requires the creation of new rules to encompass so deep changes. Also we refer to the legal framework on which to base public action today and the slowness of the established legal mechanisms to adapt it to the changing context. These seem to be very important locking elements for e-government projects.
\end{abstract}

Keywords: Electronic Government, e-Administration, e-Services, Modernizing Government

1 Ing. Electrónico, Universidad Ricardo Palma - Lima Mg. Tecnologías de la Información, UPM - Madrid Candidato a $\mathrm{PhD}$. Derecho, UNED - Madrid Consultor independiente para empresas públicas y privadas.

E-mail: jorgeluislopezc@hotmail.com Teléfono: 981-511524 


\section{EL GOBIERNO ELECTRÓNICOY LOS SERVICIOS PÚBLICOS}

En los últimos años, asistimos a importantes cambios en los modos de relación en el mundo, básicamente, por dos elementos que están determinado cambios decisivos en las empresas y en las administraciones públicas: la globalización y las tecnologías de la información y de las comunicaciones (TIC). El uso de las TIC permite que el Gobierno sea más eficiente, lo que propicia que se brinden servicios de más calidad y de fácil acceso. Asimismo, las TIC estimulan la actividad económica, sirven de apoyo en la difusión de programas y rendición de cuentas y son guía de autogestión al ciudadano. También, incrementan la comunicación tanto al interior como al exterior del Gobierno, generan certidumbre y confianza en él, informan a la población de las decisiones más importantes y crean un ambiente que facilita la participación.

Los Gobiernos juegan cada vez más un papel principal en demostrar las ventajas de la provisión de servicios electrónicos en los que es fundamental el mejoramiento del enfoque al cliente y los servicios, concentrando recursos en áreas críticas y esenciales, aumentando la competitividad en el mercado; es así que las TIC pueden ayudar a los Gobiernos a enfrentarse a los numerosos retos que se les presenta. Sin embargo, no hay que centrarse solo en las TIC, sino también en la utilización de estas tecnologías junto con un cambio organizativo con nuevos métodos para mejorar los servicios públicos, los procesos democráticos y las políticas públicas.

El gobierno electrónico (del inglés e-Government) consiste en el uso de tecnologías de la información y el conocimiento en los procesos internos de gobierno, en la entrega de los productos y servicios del Estado tanto a los ciudadanos como a la industria. Muchas de las tecnologías involucradas y sus implementaciones son las mismas o similares a aquellas correspondientes al sector privado del comercio electrónico (o e-Business), mientras que otras son específicas o únicas en relación con las necesidades del Gobierno. Los autores Criado y Ramilo (2001), definen el gobierno electrónico como:

«[...] la adopción de las TIC por las administraciones públicas, como diferentes vías a través de las que se conectan e interactúan con otras organizaciones y personas, especialmente mediante sus páginas Web, pero también a través del correo electrónico y otras herramientas como el teléfono móvil, los PDA, la vídeo conferencia, intranet ${ }^{2}$, extranet ${ }^{3}$, el cable, las ondas de radio, o el satélite.»

Como hemos señalado líneas arriba, el gobierno electrónico supone un campo de actividades del Estado más amplio que el de la Administración. El uso de las TIC al que nos referiremos normalmente alude a la Administración, por ser esta el área que mayor volumen de relaciones establece con los ciudadanos y por ser la que ha iniciado la regulación de la implementación de las TIC. En efecto, se entiende la doctrina de la e-Administración ${ }^{4}$ como el uso de las tecnologías de la información y las comunicaciones en las

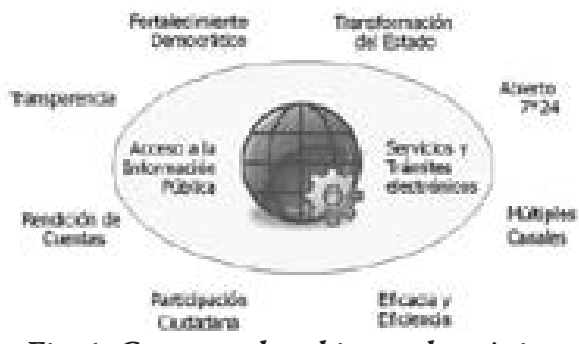

Fig. 1. Concepto de gobierno electrónico. Recuperado el 11 de julio de 2016 de http:/lagesic. gub.uy/innovaportal/v/163/1/agesic/gobierno_electronico_.html

2 Una Intranet es una red de ordenadores privados que utiliza tecnología Internet para compartir, dentro de una organización, parte de sus sistemas de información.

3 Una Extranet es una red privada que utiliza tecnología Internet y protocolos de comunicaciones para compartir, de forma segura, parte de la información propia de una organización con proveedores, compradores, socios, clientes o cualquier otro negocio u organización.

4 Administración electrónica, gobierno electrónico o e-gobierno. 
Administraciones públicas combinado con cambios organizativos y nuevas aptitudes, con el fin de mejorar los servicios públicos y los procesos democráticos y reforzar el apoyo a las políticas públicas. Pero no debe limitarse lo aquí señalado exclusivamente a esta, ya que el uso de las TIC abarca, como hemos señalado, a la totalidad de organismos de los diferentes poderes públicos cuando se relacionan entre sí o con el ciudadano. Por tanto, las ideas referidas a la administración se extienden a los demás organismos del Estado; es así que el uso de los actuales mecanismos tecnológicos de información y comunicación permiten manejar un alto volumen de información y constituyen un fundamental recurso para que el Gobierno pueda cumplir sus funciones con una productividad muy alta, empleando recursos de costo comparativamente muy bajos.

Una ventaja fundamental la constituye la conveniencia para el ciudadano de relacionarse con la Administración en ausencia, es decir, que no tienen que estar simultáneamente presentes en tiempo y lugar, el Gobierno y el ciudadano. Esto libera al particular de los estrechos horarios de atención a la ciudadanía y al Estado de mantener funcionarios apostados en instalaciones para atenderlos. Se posibilita, así, a la Administración atender a un alto volumen de ciudadanos y hacerlo las veinticuatro horas del día, todos los días del año. Los costos involucrados para mantener las TIC son bajos si los comparamos con los costos involucrados en la dotación de instalaciones físicas y de funcionarios (ver figura 1).

De acuerdo con la compañía IBM ${ }^{5}$, el gobierno electrónico consiste en interconectar horizontal -en un mismo nivel- y no verticalmente a todos los servicios y departamentos del Gobierno para mejorar sustancialmente la calidad en la entrega de servicios, con el consiguiente beneficio para la ciudadanía y las empresas. Tradicionalmente, las personas debían acudir a cuatro o cinco oficinas o departamentos de gobierno para resolver un permiso o patente, y tardaban meses e incluso años para obtener lo que buscaban; en cambio ahora todo se resuelve, como máximo, en días, y a veces horas.

Estas definiciones revelan una idea de gestión pública en que la tecnología emerge como el componente fundamental para la transformación del Estado. Sin embargo, la transformación de un aparato estatal no pasa exclusivamente por la implantación de las TIC, en particular porque, en el proceso de transformación de una estructura de tales características, el ciudadano tiene un papel preponderante y ve en las tecnologías de la información un medio ágil, efectivo y transparente para la participación ciudadana.

El gobierno electrónico, como transformación de todo un sistema de gobierno, se entiende como un cambio de paradigma en la gestión gubernamental, en un concepto de gestión que fusiona la utilización intensiva de las TIC con modalidades de gestión, planificación y administración. Bajo este punto de vista, el e-Gobierno basa y fundamenta su aplicación en la Administración pública, teniendo como objetivo principal contribuir al uso de las TIC para mejorar los servicios e información ofrecida a los ciudadanos y organizaciones, simplificar los procesos de soporte institucional y facilitar la creación de canales que permitan aumentar la transparencia y la participación ciudadana que son de especial interés cuando estas se hallan en el foco de exigencias por parte de la población en general. Todo esto haciendo énfasis en la idea de que este conjunto de iniciativas del Estado encaminadas a implantar el uso intensivo de las TIC en las actividades públicas aportan al proceso de institucionalización, estandarización, democratización, modernización, transparencia, eficiencia y, sobre todo, al cambio de mentalidad de los actores involucrados en la aspiración de una sociedad tecnológicamente avanzada como instrumento que coloque a un país en una mejor posición para su verdadero y concreto salto hacia un mejor desarrollo.

La necesidad de los Gobiernos en el mundo por agilizar, optimizar, flexibilizar, transparentar y abaratar procesos o actividades del sistema público ha motivado a utilizar en forma acelerada y sustancial las TIC. Una de las formas de este cambio los constituye el desarrollo de aplicaciones cada vez

5 Empresa Multinacional Americana de Tecnología y Consultoría Informática. 
más complejas, necesariamente apoyadas en arquitecturas dedicadas a las telecomunicaciones, especialmente diseñadas para trabajar de la manera más óptima posible, integrando sistemas, utilizando las mejores herramientas de gestión y desarrollando modelos y arquitecturas adecuadas a las necesidades del Gobierno. De este modo, se busca crear plataformas compatibles que resuelvan temas como la interoperabilidad, la compatibilidad, la accesibilidad, la seguridad, entre otros.

Los beneficios de la administración electrónica pueden ir mucho más allá de los primeros logros conseguidos por los servicios públicos en línea. Para ello, es imprescindible que la organización y las técnicas del sector público se centren en el usuario, de modo que la tecnología esté al servicio de las personas. No obstante, existen muchas barreras que hay que superar, y también se necesitan inversiones considerables. Los procesos de cambio en el ámbito de la organización y la cultura requieren un tiempo: pueden pasar muchos años antes de que la inversión en TIC, la organización y las técnicas produzcan beneficios.

Estratégicamente, la implementación de una nueva gestión pública pasa por saber cómo impulsar, en el ámbito interno de las administraciones públicas, las nuevas tecnologías de la información en un contexto de administración relacional y de gobierno multi-nivel en el que organizaciones con reducidas capacidades técnicas — por ejemplo, una parte importante de la administración local— tiene la responsabilidad de dirigir o gestionar una parte de los servicios básicos de la población.

Al facilitar las TIC la comunicación con los ciudadanos, la administración podrá informar con precisión y claridad el estado de los trámites y solicitudes que se gestionan a las personas, lo que trae como resultado una administración más transparente. En una sociedad que se proclama participativa, resulta esencial que los ciudadanos puedan estar informados sobre las tareas que lleva a cabo la administración. En principio, debe garantizarse este acceso con la única limitación del resguardo de otros derechos igualmente protegidos, como la intimidad, el derecho al honor, a la protección de datos personales, la seguridad del Estado, etc. Por otro lado, el control y las fiscalizaciones internas resultarán mucho más fáciles si se han implementado TIC, ya que, por estar digitalizada, la información puede reportarse, relacionarse y analizarse con mucho más detenimiento (ver figura 2).

Acertadamente, el gobierno electrónico es un modelo de gestión pública que se fundamenta en el uso intensivo de las TIC para proveer medios ágiles, confiables, efectivos y eficaces de información, comunicación y participación de los ciudadanos. Asimismo, garantiza la prestación segura y directa de servicios, y tiene como objetivo fundamental transformar al Estado como resultado de las mejoras de los procesos y el aumento de la eficiencia y transparencia en el poder público generado por el uso de estas tecnologías. De igual forma, el gobierno electrónico está estrechamente relacionado con los procesos y estructuras creados para la oferta electrónica de los servicios gubernamentales, y depende funcionalmente de muchas aristas o ramificaciones, como el compromiso de los gerentes públicos, la dinámica política, los servicios civiles y las funciones parlamentarias y judiciales. Se requiere que el Gobierno muestre apertura a un modelo de prestación de servicios bastante complejo en el que interactúan tanto los niveles como las aristas de su propio sistema. Así, uno de los principales objetivos del gobierno electrónico es mejorar la posición del Estado frente a la adopción de las TIC y a las políticas de Estado alineadas al plan de desarrollo de la sociedad de la información, justicia social, inclusión social, y distribución de la riqueza.

Implementar un gobierno electrónico no es automatizar todos los procedimientos que se hacen manualmente. Se trata de determinar si es necesario agregar, eliminar o reformular los procesos en base a los lineamientos que tenga la planificación estratégica de la organización. Sin una buena planificación, es decir, sin saber hacia dónde queremos llegar, no se puede desarrollar un gobierno electrónico exitoso con enfoque nacional y orientado a los ciudadanos, empresas, empleados y el propio Estado. Por tanto, es prioritario abordar el problema de la falta de una estrategia nacional de gobierno electrónico, el mismo que debe estar alineado a un Plan de Desarrollo de la Sociedad de la Información. 


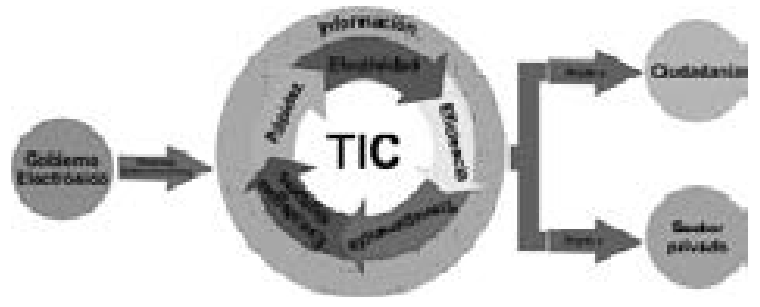

Fig. 2. Gobierno electrónico.

Recuperado el 11 de julio de 2016 de http://www.udgvirtual.udg.mx/paakat/index.php/paakat/article/view/253/376

Una de las tareas más complejas consiste en medir adecuadamente el desempeño de estas políticas de gobierno electrónico. La clave está, entonces, en la implementación de programas y políticas más eficaces. En un gran número de casos, mejorar la calidad de los servicios o hacer más eficientes las operaciones gubernamentales son solo pasos intermedios, pues los objetivos más importantes del gobierno electrónico tienen que ver con lograr políticas públicas y programas gubernamentales en forma eficiente, eficaz y transparente. Este beneficio que presta el gobierno electrónico es, quizás, uno de los más importantes, pero, al mismo tiempo, uno de los más difíciles de medir, pues supone la existencia de buenos indicadores de desempeńo para la política o un determinado programa objetivo de gobierno, como los de salud, educación, reducción de la pobreza, etc.

Un elemento estratégico para el desarrollo de los países radica en la eficiencia con que se llevan a cabo los servicios que ofrece el Gobierno a los diferentes sectores de la sociedad y su capacidad de interacción con los ciudadanos. Los países desarrollados, para mejorar los procesos internos de toma de decisiones y la relación con la ciudadanía, están resolviendo buena parte de sus problemas de funcionamiento mediante la incorporación masiva de las nuevas tecnologías de la información y comunicaciones.

La corta historia del propio concepto de gobierno electrónico produce una relativa incertidumbre y ambigüedad sobre cuáles tienen que ser las metas y los objetivos que deben alcanzarse. A falta de una experiencia más dilatada y de referentes internacionales claros, muchas de las iniciativas actuales de gobierno electrónico se dirigen principalmente a mejorar la relación con los ciudadanos mediante la prestación de servicios. En este contexto, los parámetros de evaluación del éxito de los proyectos parecen restringirse a la cantidad de servicios en línea que una administración determinada ofrece. En definitiva, se ha consolidado la idea de que a más servicios en línea, más modernización en una administración.

\section{DEL MARCO LEGAL ${ }^{6}$}

Otro aspecto destacable hace referencia al marco jurídico en el que debe basarse la acción pública en la actualidad y la lentitud de los mecanismos legales establecidos para su adecuación al contexto cambiante. Estos parecen ser elementos muy importantes de bloqueo para los proyectos de gobierno electrónico.

La sociedad de la información, una sociedad donde las tecnologías de la información y las comunicaciones alcanzan a las personas, las empresas y al Estado, y pautan nuevas formas de relación, además de un nuevo modelo de desarrollo, lo que demanda es la creación de nuevas reglas para acompasar tan profundos cambios.

El desarrollo de un nuevo marco jurídico es, por tanto, esencial para brindar garantías, tutelar los derechos de los ciudadanos y establecer reglas claras en las relaciones que establecen los distintos actores sociales. En particular, las relaciones que establecen las personas y las empresas con el Estado se desarrollan en el ámbito del gobierno electrónico. Cuando las actividades tradicionales se trasladan o

6 Ver Arcila, C. y De la Barra, R. (2009). 
efectúan por medios electrónicos, es necesario crear confianza y garantizar al usuario que estas actividades tengan idéntica o superior fiabilidad. Para ello, se ha trabajado en un nuevo marco jurídico que, en primer lugar, protege los datos personales y otorga a las personas el control sobre ellos y, en segundo lugar, les confiere validez y eficacia jurídica a los documentos electrónicos y a las firmas electrónicas.

Una segunda esfera de acción es la vinculada a la gestión del Estado, la que debe ser más eficiente y transparente para acompañar el nuevo modelo de desarrollo. En tal sentido, se aprobaron normas relativas al acceso de la información pública y normas que se relacionan con la gestión de los activos de información en poder del Estado. Entre ellas se encuentran las medidas de seguridad necesarias, un marco de referencia para el gobierno electrónico y otros instrumentos normativos referidos al desempeño de la Administración. Muchas de estas iniciativas, que responden a políticas públicas en la materia, requieren su institucionalización para implementarlas.

El presente artículo presenta una revisión documental de los principales textos legales que amparan el funcionamiento del gobierno electrónico en Espańa. Los resultados sugieren que existen dos grandes bloques legales que sustentan el uso de las TIC por el Gobierno, y tienen que ver, por un lado, con aquellas dirigidas a dotar de eficacia jurídica a las manifestaciones de voluntad por vía electrónica y, por otro, con aquellas pensadas para conseguir eficiencia en el cumplimiento de las funciones del Estado.

El análisis de los distintos instrumentos que regulan la implementación de las TIC revela que Espańa cuenta con una base jurídica sólida y avanzada, por lo que la mayoría de las deficiencias que existen se deben más bien a la implementación. Se adopta un concepto amplio de gobierno electrónico, expandiéndolo a todas las ramas del poder público en tanto y en cuanto sus órganos se relacionen con el ciudadano o entre sí. Por último, se hace un llamado al poder judicial de manera de que se incorpore de forma plena las ventajas de los recursos electrónicos a los procesos judiciales.

Entre las normas jurídicas que regulan el uso de las tecnologías de información y las comunicaciones (TIC) aplicables al desarrollo del llamado gobierno electrónico, pueden distinguirse las normas contenidas fundamentalmente en la Ley Orgánica 15/1999, de 13 de diciembre, de Protección de Datos de Carácter Personal (LOPD), ley que tiene por objeto garantizar y proteger, en lo que concierne al tratamiento de los datos personales, las libertades públicas y los derechos fundamentales de las personas físicas, y especialmente de su honor, intimidad y privacidad personal y familiar. Por otro lado, existe un conglomerado de distintos instrumentos que establecen el uso de las TIC como herramienta indispensable para la consecución de eficiencia en el cumplimiento de las diversas funciones públicas atribuidas a los distintos organismos del Estado.

Identificamos los principales textos legales que amparan el funcionamiento del gobierno electrónico en España, determinando las carencias y dificultades del desarrollo de este sistema desde una perspectiva legal, a saber:

- La Ley Orgánica 15-1999, de 13 de diciembre, de Protección de Datos de Carácter Personal.

- La Ley 34/2002 de Servicios de la Sociedad de la Información y de Comercio Electrónico.

- $\quad$ La Ley 32/2003, de 3 de noviembre, General de Telecomunicaciones.

- La Ley 59/2003, de 19 de diciembre de Firma Electrónica.

- La Ley 11/2007, de 22 junio, de Acceso Electrónico de los Ciudadanos a los Servicios Públicos.

Las TIC constituyen para el ciudadano un mecanismo fácil y sencillo de acceder a la información cuando esta se encuentra en formato electrónico. La Ley 11-2007, de 22 junio, de Acceso Electrónico de los Ciudadanos a los Servicios Públicos, que en su artículo primero, reconoce el derecho de los ciudadanos a relacionarse con las Administraciones públicas por medios electrónicos y regula los aspectos básicos de la utilización de las tecnologías de la información en la actividad administrativa, en las relaciones entre las Administraciones públicas, así como en las relaciones de los ciudadanos con las mismas con la finalidad de garantizar sus derechos, nos dice que utilizarán las tecnologías de la información 
de acuerdo con lo dispuesto en la presente ley, asegurando la disponibilidad, el acceso, la integridad, la autenticidad, la confidencialidad y la conservación de los datos, informaciones y servicios que gestionen en el ejercicio de sus competencias.

El artículo 103 de la Constitución ${ }^{7}$ señala que la Administración pública sirve con objetividad a los intereses generales y actúa de acuerdo con los principios de eficacia, jerarquía, descentralización, desconcentración y coordinación, con sometimiento pleno a la ley y al Derecho. Entendiendo a las TIC como mecanismos que mejoran el rendimiento de estos elementos, podemos concluir que su incorporación en la Administración, y en general en cualquier herramienta que catalice estos principios, es un mandato del constituyente. De allí que las TIC se vuelvan aplicables a las demás funciones que realiza el Estado.

La Ley de Acceso Electrónico de los Ciudadanos a los Servicios Públicos y La Ley de Servicios de la Sociedad de la Información y de Comercio Electrónico establecieron la necesidad de insertar a España en la sociedad del conocimiento, para lo cual el Internet representa una herramienta invalorable; así mismo, se incentiva su uso en todos los niveles. Esta ley prevé, por su parte, desarrollar el uso y aplicación de las TIC para el mejoramiento de la gobernabilidad a partir del uso de software libre, y, en especial, menciona la necesidad de crear servicios de certificación electrónica para el Estado, lo que propicia un gobierno electrónico transaccional y centrado en el ciudadano.

Inspirada en los preceptos constitucionales que hemos mencionado, la ley para el Acceso Electrónico de los Ciudadanos a las Administraciones Públicas se justifica en la creación de un marco jurídico que facilite la extensión y utilización de estas tecnologías, donde el principal reto que tiene la implantación de las TIC en la sociedad en general y en la Administración en particular es la generación de confianza suficiente que elimine o minimice los riesgos asociados a su utilización. La desconfianza nace de la percepción, muchas veces injustificada, de una mayor fragilidad de la información en soporte electrónico, de posibles riesgos de pérdida de privacidad y de la escasa transparencia de estas tecnologías.

Esta ley declara, en su artículo primero, que reconoce el derecho de los ciudadanos a relacionarse con las Administraciones públicas por medios electrónicos y regula los aspectos básicos de la utilización de las tecnologías de la información en la actividad administrativa, en las relaciones entre las Administraciones públicas, así como en las relaciones de los ciudadanos con las mismas con la finalidad de garantizar sus derechos, un tratamiento común ante ellas y la validez y eficacia de la actividad administrativa en condiciones de seguridad jurídica. Asimismo, señala que las Administraciones públicas utilizarán las tecnologías de la información de acuerdo con lo dispuesto en la presente Ley, asegurando la disponibilidad, el acceso, la integridad, la autenticidad, la confidencialidad y la conservación de los datos, informaciones y servicios que gestionen en el ejercicio de sus competencias. De esta manera, la legislación ha reconocido las ventajas de las TIC y ha ordenado incorporarlas a los distintos entes gubernamentales, pese a algunas deficiencias señaladas. Es un hecho notorio que, en los últimos ańos, muchos organismos e instituciones públicas han incorporado el uso de Internet para mejorar las comunicaciones entre sí.

Las TIC constituyen una valiosa herramienta de eficiencia administrativa, y su implementación plena en la Administración pública contribuirá notablemente a superar graves deficiencias que se padecen en muchas de las relaciones administrativas que el propio Estado impone como obligatorias a sus ciudadanos. Estas deficiencias se manifiestan, muchas veces, en una insuficiencia en la debida atención al ciudadano, lo que, a su vez, produce demoras y masivas conglomeraciones en las instalaciones físicas de atención administrativa, cuya respuesta, la mayoría de las veces, consiste en el establecimiento de restricciones al acceso, horarios reducidos, e incluso la imposición de cotas de número de ciuda-

7 Título IV-Del Gobierno y de la Administraron-Constitución Española. (octubre, 2013). Video en Youtube. Recuperado el 13/07/2016 de http://www.youtube.com/watch?v=y4U656oizUs\#t=79 
danos atendidos por día — un sistema articulado mediante el reparto diario del conocido mecanismo de "números" que se entregan de madrugada para poder acceder luego en horario de oficina al órgano Administrativo respectivo-. Es así que las TIC permiten superar todas estas deficiencias a un costo muy bajo. Para lograrlo, se cuenta con una base jurídica muy nutrida y avanzada.

Hemos visto cómo la Constitución establece un mandato de eficacia, jerarquía, descentralización, desconcentración y coordinación, con sometimiento pleno a la ley y al Derecho predicable no solo de la administración, sino de todas las ramas del poder público en tanto se relacionen internamente entre sí o con el ciudadano. Se ha resuelto, en buena parte, el problema de la eficacia jurídica mediante la Ley de Firma Electrónica. Es así que los distintos entes públicos pueden entablar relaciones administrativas electrónicas con los ciudadanos, así como emitir documentos electrónicos válidamente firmados, también, de manera electrónica.

Finalmente, considerando las ventajas de la TIC, encontramos los Decretos Supremos, que ordenan a las administraciones para poder aprovecharlas e implementarlas cuanto antes, tales como La Ley de Acceso Electrónico a los Ciudadanos a los Servicios Públicos (LAECSP), la cual obligaba a las administraciones del Estado a poner todos o casi todos sus servicios en línea. Otras ramas del poder público, sobre todo la judicial, deben aprovechar estos recursos cuanto antes y de manera más profunda de lo que lo han hecho hasta ahora, y en particular respecto a su función primordial que es la jurisdiccional. Quizás por la especialidad del proceso judicial, y la envergadura de sus efectos, resulte conveniente una regulación normativa especial que permita - y a la vez ordene- a los órganos judiciales a establecer cuanto antes un verdadero proceso judicial electrónico confiable y seguro en todas sus fases, cimentado también sobre los principios de eficacia, jerarquía, descentralización, desconcentración y coordinación.

La Justicia enfrenta numerosos retos derivados de la creciente complejidad de la sociedad y de su propia organización. Al ser un área intensiva en información, puede concretar un importante salto positivo cuando se pongan en marcha los tres procesos básicos e imprescindibles para lograr un uso eficiente de las TIC en su propia transformación: el cambio tecnológico, el organizativo y el cultural.

Tres son, también, los agentes principales en el impulso de esta nueva Justicia: el Ministerio de Justicia, el Consejo General del Poder Judicial y las Comunidades Autónomas. Todos entienden la importancia del uso de las TIC. Existe un amplio consenso respecto a la modernización de la Justicia en torno a dos elementos básicos: la nueva oficina judicial y el expediente electrónico. Este cambio implicará un nuevo paradigma organizativo, más ágil y eficiente, que abrirá el camino hacia un modelo de justicia en red y a su mejora administrativa. ${ }^{8}$

La implantación de las TIC constituye una gran oportunidad para el desarrollo de una Justicia más accesible, próxima y transparente, que ofrezca nuevos canales de acceso a la información, mejor relación con los ciudadanos y un servicio de mayor calidad capaz de garantizar -con rapidez y eficacialos derechos legalmente reconocidos.

\section{CONCLUSIONES}

La administración electrónica o gobierno electrónico es la manera en que los Gobiernos emplean las nuevas tecnologías para proporcionar a las personas un mejor acceso a la información y a los servicios gubernamentales, mejorar la calidad de los servicios y dar más oportunidades para participar en los procesos y en las instituciones democráticas.

8 Organización de los Estados Americanos. FORO e-gobierno OEA. Recuperado el 13/07/2016 de http://www.suboletin. $\mathrm{com} /$ contentsoea/docs/Boletin_65/Paratenerencuenta65.htm 
El gobierno electrónico es el conjunto de iniciativas del Estado encaminadas a implantar el uso intensivo de las tecnologías de la información y de las comunicaciones (TIC) en las actividades públicas que aportan al proceso de institucionalización, estandarización, democratización, modernización, transparencia, eficiencia y, sobre todo, al cambio de mentalidad de los actores involucrados en la aspiración de una sociedad tecnológicamente avanzada, como instrumento que coloque al país en una mejor posición para su verdadero y concreto salto hacia el desarrollo.

Las nuevas tecnologías permiten una supervisión mucho más estrecha y una información más detallada sobre las operaciones y procesos internos. Al mismo tiempo, las TIC se están utilizando para respaldar nuevos métodos de seguimiento y control de los resultados de las políticas y programas gubernamentales.

España cuenta con una base jurídica sólida y avanzada, por lo que la mayoría de las deficiencias que existen se deben más bien a la implementación. Se adopta un concepto amplio de gobierno electrónico, expandiéndolo a todas las ramas del poder público en tanto y en cuanto sus órganos se relacionen con el ciudadano o entre sí.

\section{REFERENCIAS}

Arcila, C. y De la Barra, R. (2009). Aspectos legales del gobierno electrónico en Venezuela. Disponible en http://erevistas.saber.ula.ve/index.php/Disertaciones/article/view/50/67.

Bañón, R. y Carrillo, E. (2006). La nueva administración pública. Madrid: Alianza.

Bidyut Chakra B. y Bhattacharya, M. (2005). University, Administrative Change and Innovation. Oxford University.

Boletín Oficial del Estado.

_Ley 34/2002, de 11 de julio, de Servicios de la Sociedad de la Información y de Comercio Electrónico (LSSI). Recuperado el 18/06/2016 de http://www.boe.es/buscar/pdf/2002/BOE-A-2002-13758consolidado.pdf.

_Ley 32/2003, de 3 de noviembre, General de Telecomunicaciones. Recuperado el 18/06/2016 de https://www.boe.es/buscar/doc.php?id=BOE-A-2003-20253.

_Ley 59/2003, de 19 de diciembre, de Firma Electrónica. Recuperado el 18/06/2016 de http://www. boe.es/buscar/doc.php?id=BOE-A-2003-23399.

_Ley 11/2007, de 22 de junio, de Acceso Electrónico de los Ciudadanos a los Servicios Públicos. Recuperado el 18/06/2016 desde https://www.boe.es/buscar/doc.php?id=BOE-A-2007-12352.

Bonnin, C.-J. (2004). Principios de la administración pública. México: Fondo de Cultura Económica.

Criado I., Gascó, M. y Jimenez, C. E. (2010). Marco Iberoamericano de Interoperabilidad. Caracas, Venezuela: CLAD.

Dunleavy, P. y Margaretts H., (2006). Digital Era Governance: IT Corporations, the State and E-Government. Oxford University.

Gascó, M. y Roy, J. (2006). E-Government and Multi-Level Governance: A Comparative Examination of Catalonia, Spain, and Ontario, Canada. International Journal of Electronic Government Research, 2(4), pp. 57-75.

GEALC (2007). La intranet gubernamental como elemento clave de la interoperabilidad. Red de Líderes de Gobierno Electrónico de América Latina y El Caribe.

Hood C. y Margaretts, H. (2007). The Tools of Government in the Digital Age. Nueva York: Palgrave.

López, J. y Gadea, A., (2001). Una nueva administración pública. Estrategias y métodos para mejorar la calidad y la eficiencia del Gobierno. Ońati: Instituto Vasco de Administración Pública.

Olías de Lima, B. (2006). Manual de Organización Administrativa del Estado. Madrid: Síntesis. 
Ramió, C. (2001). Los problemas de la implantación de la nueva gestión pública en las administraciones latinas: modelo de Estado y cultura institucional. Revista del CLAD Reforma y Democracia, 21.

Ramió, C. (1999). Teoría de la organización y administración pública. Madrid: Editorial Tecnos.

Ramió, C. (octubre, 2005). El papel de la e-administración ante los retos de las administraciones públicas de América Latina: la e-administración y sector público en el siglo de la globalización. Documento de Formación Municipal No 94.

\section{ENLACES DE INTERÉS}

The Digital Governance Initiative. Disponible en: http://www.digitalgovernance.org [en línea] [Consulta: 02 de julio de 2016].

Biblioteca Jurídica, Análisis del Poder Político, UNAM. Disponible en http://biblio.juridicas.unam. mx/libros/6/2749/4.pdf [en línea] [Consulta: 02 de julio de 2016].

Oficina Nacional de Gobierno Electrónico e Informática. Disponible en: http://www.ongei.gob.pe/ entidad/ongei_tematicos.asp?cod_tema=5849. [en línea] [Consulta: 03 Jul. 2016].

Interoperabilidad de Gobierno Electrónico en Iberoamérica. Estudio comparativo y recomendaciones de futuro. Revista del CLAD Reforma y Democracia, 50, junio 2011. ISSN 13152378. Disponible en: http://www.scribd.com/doc/76536927/Interoperabilidad-de-Gobiernoelectronico-en-Iberoamerica-Estudio-comparativo-y-recomendaciones-de-futuro. [en línea] [Consulta: 02 Jul. 2016].

CEPAL, Libro Blanco de Interoperabilidad de Gobierno Electrónico para América Latina y Caribe, Santiago de Chile (Chile): CEPAL y Europe Aid. Documento disponible en http://www.eclac. org/ddpe/publicaciones/xml/2/37352/Libroblanco.pdf [en línea] [Consulta: 03 Jul. 2016].

Consejo Superior de Administración Electrónica del Ministerio de Administraciones Públicas, Ex Consejo Superior de Informática. Documento disponible en: http://www.csi.map.es/ , secretaria. redsara@map.es, Dic 2008. [en línea] [Consulta: 03 Jul. 2016].

Proyectos de Modernización Administrativa Local (eModel) - MAP, Octubre 2008. Documento disponible en: http://www.csi.map.es/csi/nuevo/administración_electrónica_3.htm , Dic. 2008. [en línea] [Consulta: 03 Jul. 2016].

AGPD. Ley Orgánica de Protección de Datos (LOPD). Recuperado el 18 de junio de 2016 de http:// www.agpd.es/portalwebAGPD/LaAgencia/index-ides-idphp.php.

Ley 30-1992, de 26 de noviembre, de Régimen Jurídico de las Administraciones Públicas y del Procedimiento Administrativo. Documento disponible en: https://www.boe.es/buscar/doc. php?id=BOE-A-1992-26318 . [en línea] [Consulta: 02 de julio de 2016].

Ley 30-2007, de 30 de octubre, de Contratos del Sector Público. Disponible en: https://www.boe.es/ buscar/doc.php?id=BOE-A-2007-18874. [en línea] [Consulta: 02 de julio de 2016].

Ley Orgánica 15-1999, de 13 de diciembre, de Protección de Datos de Carácter Personal. Documento disponible en: https://www.boe.es/buscar/doc.php?id=BOE-A-1999-23750 [en línea] [Consulta: 02 Jul. 2016]. 